\title{
RESONANCE CLASSES OF MEASURES
}

\author{
MARIA TORRES DE SQUIRE \\ Department of Mathematics and Statistics \\ University of Regina \\ Regina, Saskatchewan \\ S4S OA2 Canada
}

(Received September 30, 1986)

\begin{abstract}
We extend F. Holland's definition of the space of resonant classes of functions, on the real line, to the space $R\left(\Phi_{p q}\right)(1 \leqq p, q \leqq \infty)$ of resonant classes of measures, on locally compact abelian groups. We characterize this space in terms of transformable measures and establish a realationship between $R\left(\Phi_{p q}\right)$ and the set of positive defintte functions for amalgam spaces. As a consequence we answer the conjecture posed by L. Argabright and J. Gil de Lamadrid in their work on Fourier analysis of unbounded measures.
\end{abstract}

KEY WORDS AND PHRASES. Amalgam spaces, Fourier transform of unbounded measures, positive definite measures, positive definite quasimeasures, Fourier multipliers.

1980 AMS SUBJECT CLASSIFICATION CODE. 43A35, $43 \mathrm{~A} 15$.

1. INTRODUCTION.

F. Holland [1] defined the space $R\left(\Phi_{\infty q}\right)(1 \leq q \leq \infty)$ of resonance classes of functions, on the real line, relative to the space of test functions $\Phi_{\infty q}$, and proved that a function belongs to $R\left(\Phi_{\infty_{q}}\right)(2 \leqq q \leqq \infty)$ iff it is the Fourier transform of an unbounded measure [1, Theorem 6]. He also pointed out that the set $P\left(C_{c}\right)$ of positive definte functions in Cooper's sense [2] is included in $R\left(\Phi_{\infty 1}\right)[1, \S 1]$, and proved that every function in $R\left(\Phi_{\infty 1}\right)$ has the same representation in terms of unbounded measures as the functions in $P\left(C_{c}\right)$ [1, Theorems 7 and 8], [3, Theorems 4.1 and 4.2] (1n fact, as we will prove here, these representations hold for a larger class of functions and they are equivalent). These results of Holland together with Bochner's theorem on positive definite functions [4] - a function is the Fourier transform of a bounded measure iff it is a linear combination of positive definite functions - lead one to speculate that any function in $R\left(\Phi_{\infty_{q}}\right)$ is a linear combination of positive definite functions. In the present paper we respond to this conjecture in a more general setting. We define the space $R\left(\Phi_{\mathrm{pq}}\right)(1 \leqq \mathrm{p}, \mathrm{q} \leqq \infty)$ of resonance classes of measures (on locally compact abelian groups) relative to $\Phi_{p q}$, which includes $R\left(\Phi_{\infty q}\right)$ as a particular case; we characterize this space in terms of transformable measures [5], and prove that for $1 \leqq \mathrm{p} \leqq \infty$, any measure in $R\left(\Phi_{\mathrm{pq}}\right)(2 \leqq \mathrm{q} \leqq \infty)$ is a 1inear combination of positve definite functions for some amagam space $\left(L^{r}, \ell^{s}\right)[6]$, and for $1 \leq q<2$, any measure 
in $R\left(\Phi_{\mathrm{pq}}\right)$ can be approximated by linear combinations of positive definite functions for some amalgam $\left(L^{r}, \ell^{s}\right)$.

From these results we conclude that $P\left(C_{c}\right)$ is dense in $R\left(\Phi_{\infty 1}\right)$ and $\left\langle\rho\left(C_{c}\right)\right\rangle$, the space generated by the set of positive definite measures as defined in [5, 54], is dense in the space of transformable measures. This answers the conjecture posed in [5].

Throughout the whole paper G will be a locally compact abelian group with Haar measure $m$ and dual group $\Gamma$. For an element $\hat{x}$ in $\Gamma$ we write $[x, \hat{x}]$ instead of $\hat{\mathbf{x}}(\mathbf{x})(\mathbf{x} \in G)$. Given two sets $A$ and $B$ we denote by $A-B$ the set $\{x-y \mid x \in A, y \notin B\}$. For a function $f$ on $G$ we use $\tilde{f}$ to denote its involution, i.e. $\tilde{f}(x)=\overline{f(-x)}$. The space of continuos functions which vanish at infinity, with compact support, will be denoted by $C_{0}, C_{c}$, respectively, We endow $C_{c}$ with the inductive 1 imit topology, as in [5]. By a measure (on $G$ ) we will mean an element of the continuous dual of $C_{C}(G)$. We let $M$ be the space of measures on $G$.

A function $f$ belongs to $L_{10 c}^{q}(1 \leqq q \leqq \infty)$ if $f$ restricted to any compact subset of $G$, belongs to $L^{q}$, and $f$ belongs to $L_{c}^{q}(1 \leqq q \leqq \infty)$ if $f$ has compact support and belongs to $\mathrm{L}^{\mathrm{q}}$.

The amalgam spaces $\left(L^{p}, \ell^{q}\right),\left(C_{0}, \ell^{q}\right)(1 \leq p, q \leq \infty)$ and the space of measures $M_{s}(1 \leq s \leq \infty)$ will be as defined in [7]. We will make constant use of the following inclusions and inequalities proven in [7].

$$
\begin{array}{ll}
\left(L^{p}, \ell^{q_{1}}\right) \subset\left(L^{p}, \ell^{q_{2}}\right) & q_{1} \leqq q_{2} \\
\left(L^{p_{1}} \ell^{q}\right) \subset\left(L^{p_{2}}, \ell^{q}\right) & p_{1} \geqq p_{2} \\
\left(L^{p}, \ell^{q}\right) \subset L^{p} \cap L^{q} & p \geqq q \\
\|f\|_{p q_{2}} \leqq \|\left. f\right|_{p q_{1}} & q_{1} \leqq q_{2} \\
\left.|| f\left\|_{p_{1} q} \leqq\right\| f\right|_{p_{2} q} & p_{1} \geqq p_{2}
\end{array}
$$

We will assume all the results of duality and convolution product for these spaces, the Holder and Young's inequalities, and the Hausdorff-Young theorem for amalgams as given in $\left[8, \S 1, \S_{2}\right]$.

The Fourier transform (inverse of the Fourler transform) of a measure $\mu$ on $G$ (on $\Gamma$ ) will be denoted by $\rho(\stackrel{V}{\mu})$. We will denote by $\left\{e_{\alpha}\right\}$ the approximate Identity of the algebra $L^{1}(G)$ consisting of continuous functions with a fixed support and positive Fourier transform in $L^{1}(\Gamma)$.

We let $\left\{\psi_{U} \mid U\right.$ a compact neighbourhood of 0$\}$ be the family of functions $\psi_{U}$ in $A_{c}$, the space of functions in $C_{c}$ whose Fourier transform belongs to $L^{1}$, with the following properties

$\operatorname{supp} \psi_{\mathrm{U}} \subset \mathrm{U}$

$\psi_{U}=B_{U} \star \tilde{\beta}_{U}$, where $\beta_{U} \in L_{c}^{2}$

$\psi_{\mathrm{U}} \geqq 0 \quad$ and $\quad \hat{\psi}_{\mathrm{U}} \geqq 0$

$\lim \psi_{U}=1$ uniformly on any compact subset of $\mathrm{g}$

$\left\{\psi_{U}\right\}$ is an approximate identity of $L^{1}$ 
The duality between a Banach space $B$ and its Banach dual B' will be denoted by $\langle\mathrm{f}, \mathrm{F}\rangle, \mathrm{F} \in \mathrm{B}^{\prime}, \mathrm{f} \in \mathrm{B}$. As in [5] we call a measure $\mu$ on $\mathrm{G}$ transformable, if the linear space $C_{2}(G)$, generated by the set $\left\{f * \tilde{f} \mid f \in C_{c}(G)\right\}$, is included in $L^{1}(\mu)$, and there exists a measure $\hat{\rho}$ on $\Gamma$ such that $\int f * \tilde{f}(x) d \mu(x)=\int|\hat{f}|^{2}(-\hat{x}) d \hat{\mu}(\hat{x})$ for all f $\varepsilon C_{c}$. We denote by $M_{\mathrm{T}}$ the space of transformable measures.

2. POSITIVE DEFINITE MEASURES.

We follow the definition of positive definite measures given by Dupuis in [6], but using the Segal algebra $S_{0}(G)$ which is equivalent to the space of translation bounded quasimeasures [9]. The advantage is that for $\sigma \varepsilon S_{0}(G)$ ' its Fourier transform $\hat{\sigma}$ belongs to $S_{0}(\Gamma)^{\prime}[10]$ and for $f \in L^{1}$ we have, as proven in [8, §2], that

$(\sigma * f)^{\wedge}=\hat{\sigma} \hat{f}$ and $(\sigma \hat{f})^{\vee}=\stackrel{\sigma o f}{f}$

$\sigma=\sigma$.

We assume all definitions and results about the algebra $S_{0}(G)$ given in [8, 52]. From these it is not difficult to see that the Fourier transform of a transformable measure $\mu$ (considered as an element of $S_{0}(G)^{\prime}[10]$ ) corresponds to the measure $\hat{\mu}$ associated to $\mu$.

As in [10], an element $\sigma$ in $S_{0}(G)^{\prime}$ is positive, $\sigma \geqq 0$, if $f$ positive in $S_{0}(G)$ implies $\sigma(f)$ positive. In this sense a function $g$ in $S_{0}(G)^{\prime}$ is positive iff $g(x) \geq 0$ almost everywhere. Indeed, let $\mu$ be the measure $g \mathrm{dm}$ and suppose $g$ in $S_{0}(G)$ ' positive. For $\phi \in C_{c}$ positive, the function $\phi^{*} e_{\alpha}$ is a positive element of $S_{0}(G)$ and converges to $\phi$ in $C_{c}$ [5]. So we have that $\mu(\phi)=\lim \left\langle\phi^{*} e_{\alpha}, \mu\right\rangle=\lim \left\langle\phi^{*} e_{\alpha}, g\right\rangle \geqslant 0$. Hence $\mu$ is a positive measure and therefore $g(x) \geqq 0$ almost everywhere [11, Chp. III].

DEFINITION 2.1. Let $E$ be a subset of functions of $S_{0}(G)^{\prime}$. A measure $\mu$ is a positive definite measure for $E$ if

(D1) $\quad \mathrm{E} \subset \mathrm{L}^{1}(\mu)$

(D2) $\langle\mathrm{h}, \mu>\geq 0$ for all $\mathrm{h} \in \mathrm{E}$ such that $\hat{\mathrm{h}} \geqq 0$.

We write $P(E)$ to denote the set of positive definite measures for $E$, and $P(E)$ to denote the set of measurable functions in $\rho(E)$. For a set $E$ as in Definition 2.1 we denote by $\mathrm{E}^{+}$the set of functions in $\mathrm{E}$ whose Fourier transform is positive, and by $\langle\rho(E)\rangle$ the linear space generated by $\rho(E)$.

Clearly, Definition 2.1 is equivalent to Dupuis' definition of positive definite measures [6]. By $[10$, Theorem Bl] and [8, (1.9)] the set $E$ can be any amalgam space, hence any $L^{\mathrm{P}}$ space $[8,(1.4)]$.

Argabright and Gil de Lamadrid have studied the set $\rho(G)$, of measures $\mu$ such that $\langle\phi * \tilde{\phi}, \mu\rangle \geqq 0$ for all $\phi \varepsilon C_{c}$, in connection with the space of transformable measures. We use their results in [5] to prove that $\rho(G)$ is equal to $\rho\left(C_{c}\right)$.

PROPOSITION 2.2. A measure $\mu$ belongs to $\rho\left(C_{C}\right)$ iff $\langle\phi \star \tilde{\phi}, \mu\rangle \geq 0$ for all $\phi$ in $\mathrm{C}_{\mathrm{c}^{\circ}}$

PROOF. The inclusion $\rho\left(C_{c}\right) \subset \rho(G)$ is clear. Take $\mu \varepsilon \rho(G)$. Since $c_{c}$ is included in $M_{\mathrm{T}}$ [5, Theorem 2.2] we have that a function $\phi$ in $\mathrm{C}_{\mathrm{c}}^{+}$is a continuous positive definite function [5, Theorem 4.1], so $\langle\phi, \mu\rangle \geqq 0$ by [5, Corollary 4.2] and therefore $\mu$ satisfies condition (D2). 
REMARK 2.3. It is clear that if $E_{1} \subset E_{2}\left(E_{1}, E_{2}\right.$ as in Definition 2.1), then $\rho\left(E_{1}\right) \subset \rho\left(E_{2}\right)$, so by [5, Theorem 4.1], if $c_{c}$ is a subset of $E$, then $\rho(E) \subset M_{T}$.

Dupuis defined the set $\rho$ of positive definite quasimeasures to be the set of all quasimeasures $\sigma$ such that $\langle\phi, \sigma\rangle \geqq 0$ for all $\phi$ in $A_{c}{ }^{+}$, and characterized it as the set $\left\{\hat{\mu} \mid \mu \varepsilon, M_{\infty}\right\} \quad[6$, Proposition II].

As in [6, Proposition II] we use the following lemma to prove Theorem 2.5.

LEMMA 2.4 . Let $A$ be any of the amalgam spaces $\left(L^{p}, \ell^{q}\right),\left(C_{0}, l^{s}\right)$ $(1 \leqq p, q<\infty, 1 \leqq s \leqq \infty)$. If $f \in A^{+}$, then there exists a net $\left\{f_{n}\right\}$ in $A_{c}^{+}$such that $\lim \mathrm{f}_{\mathrm{n}}=\mathrm{f}$ in $\mathrm{A}$.

PROOF. Since $\left\{\psi_{U}\right\} \subset \mathrm{C}_{c}$, we have by [8, Theorem 1.6] that the net $\left\{f \psi_{U}{ }^{*} e_{\alpha}\right\}$ is included in $c_{c}$. Thus by (2.1) its Fourier transform $\left(f \psi_{U}{ }_{e}\right)^{\wedge}=\left(f \psi_{U}\right) \wedge \hat{e}_{\alpha}=\left(\hat{f} \star \hat{\psi}_{U}\right) \hat{e}_{\alpha}$ is positive $\left[8,(2.5),(2.6]\right.$, and since $e_{\alpha}$ belongs to $L^{1}$, the net $\left\{f \psi_{U}{ }^{*} e_{\alpha}\right\}$ is included in $A_{c}$. Finally as in [6, Theorem III c)] $\lim f \psi_{U}{ }^{*} e_{\alpha}=f$ in A (see also [8, Proposition 1.8]).

THEOREM 2.5. Let $1 \leqq \mathrm{p}, \mathrm{q}<\infty$. Then $\mathrm{P}\left(\mathrm{L}^{\mathrm{p}}, \ell^{\mathrm{q}}\right)$ is equal to $\left(\mathrm{L}^{\mathrm{p}^{\prime}}, \ell^{\mathrm{q}^{\prime}}\right) \cap \rho$. PROOF. If $\mu$ is in $\left(L^{p^{\prime}}, \ell^{q^{\prime}}\right) \cap \rho$ and $f$ is an element of $\left(L^{p}, \ell^{q}\right)^{+}$, then there is a net $\left\{f_{n}\right\}$ as stated in the previous lemma, so by [7, Theorem 3.2] we have that $\langle\mathrm{f}, \mu\rangle=1 \mathrm{im}\left\langle\mathrm{f}_{\mathrm{n}}, \mu\right\rangle \geqslant U$. Therefore $\mu \varepsilon P\left(\mathrm{~L}^{\mathrm{p}}, \ell^{\mathrm{q}}\right)$. The other inclusion follows from [6, Proposition IV].

REMARKS 2.6. From (2.2) and Theorem 2.5 we have that if $\nu$ is a positive measure in $M_{\infty}$ such that $\hat{v} \varepsilon\left(L^{p^{\prime}}, \ell^{q^{\prime}}\right)(1 \leqq p, q<\infty)$, then $\hat{v}$ belongs to $P\left(L^{p}, \ell^{q}\right)$.

3. RESONANCE CLASSES OF MEASURES.

Bertrandias and Dupuis [12] defined the space $\Phi_{p q}(1 \leqq p, q \leqq \infty)$ of test functions on locally compact abelian groups based on Holland's definition of the space $\Phi_{\infty}$ $(1 \leqq \mathrm{q} \leqq \infty)$ for the real line.

DEFINITION 3.1. Let $1 \leqq p, q \leqq \infty$. The space $\Phi_{p g}(G)=\Phi_{p q}$ consists of all functions $\phi$ in $C_{c}(G)$ such that $\hat{\phi}$ belongs to $\left(C_{0}, \ell\right)(\Gamma)$, endowed with the norm $\phi \longmapsto|| \hat{\phi}||$

The space $\Phi_{\infty 1}$, used by Bertrandias and Dupuis for their definition of the Fourier transform is equal (as a set) to $A_{c}[13],[9]$. Hence the space $C_{2}(G)$ is included in $\Phi_{\infty 1}(G)[14]$. We will use this in Theorem 4.2.

REMARK 3.2. i) As sets $\Phi_{\mathrm{pq}}=\Phi_{\mathrm{rq}}$ for $1 \leqq \mathrm{p}, \mathrm{q}, \mathrm{r} \leqq{ }^{\infty}$, and $\Phi_{\mathrm{pq}}=\mathrm{C}_{\mathrm{c}}$ for $1 \leqq \mathrm{p} \leqq \infty, 2 \leqq \mathrm{q} \leqq \infty$ by the Hausdorff-Young theorem.

ii) A linear functional $\mathrm{T}$ on $\Phi_{\mathrm{pq}}(\mathrm{G})(1 \leq \mathrm{p}, \mathrm{q} \leq \infty)$ is continuous iff there is a unique measure $\mu$ in $M_{q^{\prime}}(\Gamma)$ if $p=\infty$, in $\left(L^{p^{\prime}}, \ell^{q^{\top}}\right)(\Gamma)$ if $p<\infty$, such that

$$
T(\phi)=\int \Gamma \hat{\phi}(-\hat{x}) d \mu(\hat{x})
$$

for $\left.\phi \varepsilon \Phi_{\mathrm{pq}}[2, \S 2 \mathrm{c})\right]$.

The next definition extends Holland's definition of the space of resonance classes of functions $[1, \S 5]$.

DEFINITION 3.3. Let $1 \leqq \mathrm{p}, \mathrm{q} \leqq \infty$. A measure $\mu$ on $\mathrm{G}$ is resonant relative to $\Phi_{\mathrm{pq}}(\mathrm{G})$ if

$(\mathrm{R} 1) \Phi_{\mathrm{pq}} \subset \mathrm{L}^{1}(\mu)$ 
(R2) The map $\phi \longmapsto<\phi, \mu>$ is continuous on $\Phi_{\mathrm{pq}}$, i.e. there exists a constant C such that $\mid\left\langle\phi, \mu>|\leqq c||\hat{\phi}|_{\mathrm{pq}}\right.$.

We denote by $R\left(\Phi_{\mathrm{pq}}\right)$ the space of resonance classes of measures relative to $\Phi_{\mathrm{pq}}$, and by $R\left(\Phi_{\mathrm{pq}}\right)$ the space of functions in $R\left(\Phi_{\mathrm{pq}}\right)$. By (1.1) it is clear that $R\left(\Phi_{\mathrm{pq}}\right)$ is included in $R\left(\Phi_{\mathrm{rs}}\right)$ if $1 \leqq \mathrm{~s} \leqq \mathrm{q} \leqq \infty, 1 \leqq \mathrm{p}, \mathrm{r} \leqq \infty$.

H. Feichtinger has given a more general definition of resonance classes of functions relative to the space $\Phi_{B}$, where $B$ is a Banach space of functions containing $S_{0}(G)$ as a dense subspace (private communication).

THEOREM 3.4. i) A function $f$ satisfies (R1) iff $f \in L_{10 c}^{1}$

ii) A measure $\mu$ belongs to $R\left(\Phi_{\mathrm{pq}}\right)(1 \leqq \mathrm{p}, \mathrm{q} \leqq \infty)$ iff $\mu \in M_{\mathrm{T}}$ and $\hat{\mu} \varepsilon \mathrm{M}_{\mathrm{q}^{\prime}}(\Gamma)$ if $\mathrm{p}=\infty, \hat{\mu} \in\left(\mathrm{L}^{\mathrm{p}^{\prime}}, \ell^{\mathrm{q}^{\prime}}\right)(\Gamma)$ if $\mathrm{p}<\infty$.

iii) For $2 \leqq \mathrm{q} \leqq \infty$ we have that $R\left(\Phi_{\mathrm{pq}}\right) \subset\left(\mathrm{L}^{\mathrm{q}}, \ell^{\mathrm{p}}\right)$ if $2 \leqq \mathrm{p} \leqq \infty$, and $R\left(\Phi_{\mathrm{pq}}\right) \subset\left(\mathrm{L}^{\mathrm{q}}, \ell^{2}\right)$ if $1 \leqq \mathrm{p} \leqq 2$.

PROOF. The proof of $i)$ is similar to the real case [1, Theorem 1] using [7, Theorem 3.1].

To prove ii) take $\mu$ in $R\left(\Phi_{\mathrm{pq}}\right)$ and set the map $\mathrm{T}(\phi)=\langle\phi, \mu\rangle$ on $\Phi_{\mathrm{pq}}$. If $\mathrm{T}$ is continuous, then by Remark 3.2 there exists a measure $\nu$ as stated in the theorem such that $\int \hat{\phi}(-\hat{x}) d \nu(\hat{x})=\int \phi(x) d \mu(x)$. Since $C_{2}(G) \subset \Phi_{\infty 1}(G)$ we conclude that $\mu \varepsilon M_{T}$ and $\hat{\mu}=\nu$. Conversely if $\mu \varepsilon M_{T}$ and $\hat{\mu} \varepsilon M_{q^{\prime}}(\Gamma)$, then for $\phi \varepsilon \Phi_{\infty}$, $\hat{\phi} \varepsilon L^{1}(\Gamma)$ [8, Theorem 1.4]. Hence by [5, Corollary 3.1] and Young's inequality we have that $\left|\int \phi(x) d \mu(x)\right|=|\delta \hat{\phi}(-\hat{x}) d \hat{\mu}(\hat{x})| \leqq\left.|| \hat{\mu}\right|_{q^{\prime}}|| \hat{\phi}||_{\infty_{q}}$. Therefore $\mu \varepsilon R\left(\Phi_{p q}\right)$. The proof for $p$ finite is the same.

Part iii) follows from the Hausdorff-Young theorem and the fact that the spaces $\left(\mathrm{L}^{\mathrm{p}^{\prime}}, \ell^{\mathrm{q}^{\prime}}\right)$ and $\mathrm{M}_{\mathrm{q}^{\prime}}(2 \leqq \mathrm{q} \leqq \infty)$ are included in $M_{\mathrm{T}}$ [15, Remark 6.25].

We conclude from Theorem 3.4 [5, Theorem 2.5] that $R\left(\Phi_{p q}\right)=R\left(\Phi_{p q}\right)$ for $1 \leq p \leq \infty$, $2 \leqq \mathrm{q} \leqq \infty, \quad \mathrm{R}\left(\Phi_{\infty 1}\right)=\mathrm{L}_{10 \mathrm{c}}^{1} \cap M_{\mathrm{T}}$ and $R\left(\Phi_{\infty 1}\right)=M_{\mathrm{T}}$.

The following corollary is easily deduced from the previous theorem and the Hausdorff-Young theorem.

COROLlaRY 3.5 . Let $1 \leqq p \leqq \infty, 2 \leqq q \leqq \infty$. A function f belongs to $R\left(\Phi_{p q}\right)$ iff there exists a unique $\mu \in \mathrm{M}_{q^{\prime}}(\Gamma)$ if $p=\infty, \mu \varepsilon\left(L^{p^{\prime}}, l^{q^{\prime}}\right)(\Gamma)$ if $p<\infty$, such that $\mathbf{f}=\stackrel{v}{\mathrm{r}}$.

Since $\Phi_{\infty \mathrm{q}}=\mathrm{C}_{\mathrm{c}}$ if $\mathrm{q} \geqq 2$, Corollary 3.5 includes the results of Eberlein [16, Theorem 1] (with $p=q=\infty$ ) and Stewart [7, Theorem 4.4] (with $p=\infty$ ) as special cases.

We observe that the measure $\mu$ in Corollary 3.5 is precisely the Fourier transform of $\mathrm{f}$.

We now establish the relationship between $R\left(\Phi_{p q}\right)(2 \leqq q \leqq \infty)$ and the set of positive definite functions.

PROPOSITION 3.6. Let $2 \leqq q \leqq \infty$. The space $R\left(\Phi_{\mathrm{Pq}}\right)$ is a subspace of $\left\langle P\left(L^{P^{\prime}}, \ell^{q^{\prime}}\right)>\right.$ if $2 \leq \mathrm{p} \leq \infty$, of $\left\langle\mathrm{P}\left(\mathrm{L}^{\mathrm{q}^{\prime}}, \mathrm{l}^{2}\right)>\right.$ if $1 \leq \mathrm{p} \leq 2$.

PROOF. If $f \in R\left(\Phi_{\infty}\right)$, then $f=O_{1}-O_{2}+i\left(O_{3}-O_{4}\right)$ where $\nu_{j} \geq 0, \nu_{j} \varepsilon M_{q}$ if $p=\infty$, and $\nu_{j} \in\left(L^{p^{\prime}}, \ell^{q^{\prime}}\right)$ if $p<\infty \quad(j=1, \ldots, 4)$ [11, Chp. III]. By the HausdorffYoung theorem $\hat{\nu}_{j} \in\left(L^{q}, \ell^{p}\right)$ if $2 \leqq p \leqq \infty$, and $\nu_{j} \varepsilon\left(L^{q}, \ell^{2}\right)$ if $1 \leqq p \leqq 2$ (j=1,. $., 4)$. So by Remark 2.6 we conclude that $D_{j}(j=1, \ldots, 4)$ belongs to $P\left(L^{q^{\prime}}, \ell^{p^{\prime}}\right)$ if $2 \leqq p \leqq \infty$, to $P\left(L^{q^{\prime}}, \ell^{2}\right)$ if $1 \leqq p \leqq 2$. 
COROLLARY 3.7. $\left\langle\mathrm{P}\left(\mathrm{L}^{2}, \ell^{1}\right)\right\rangle=\mathrm{R}\left(\Phi_{\infty 2}\right)$ and $\left\langle\mathrm{P}\left(\mathrm{L}^{2}\right)\right\rangle=\mathrm{R}\left(\Phi_{22}\right)$.

PROOF. By Theorem 2.5, $\mathrm{P}\left(\mathrm{L}^{2}, \ell^{1}\right) \subset\left(\mathrm{L}^{2}, \ell^{\infty}\right)$ and $\mathrm{P}\left(\mathrm{L}^{2}\right) \subset \mathrm{L}^{2}$, hence if $\mathrm{f}$ is in $P\left(L^{2}, \ell^{1}\right)$, then by [6, Theorem II] $\hat{f} \in M_{2}$, so by Remark 2.3 and Theorem 3.4 we conc= lude that $f \in R\left(\Phi_{\infty 2}\right)$. Similarly if $f \in P\left(L^{2}\right)$, then by Theorem $3,4, \hat{f} \varepsilon R\left(\Phi_{22}\right)$ and the equalities follow from Proposition 3.6.

For the remaining cases, that is, for $2 \leqq \mathrm{p} \leqq \infty, 2<\mathrm{q} \leqq \infty^{\infty} ; 1 \leqq \mathrm{p}<2,2 \leqq \mathrm{q} \leqq{ }^{\infty}$; and $q=2,2<p<\infty$, the inclusions in Proposition 3.6 are proper because the Fourier transforms on $\left(\mathrm{L}^{\mathrm{r}}, \ell^{\mathrm{s}}\right)(1<\mathrm{r}<2,1 \leqq \mathrm{~s} \leqq 2)$, on $\mathrm{M}_{\mathrm{s}}(1 \leqq \mathrm{~s} \leqq 2)$, and on ( ${ }^{2}$, $\left.\ell^{\mathrm{s}}\right)$ $(1 \leqq s<2)$ are not onto [17, Corollary 6.3]. Indeed if $2 \leqq p \leqq \infty$ and $2<q \leqq \infty$, then there exists $f \in\left(L^{q}, \ell^{p}\right)$ such that $f \neq \hat{h}$ for all $h \in\left(L^{p^{\prime}}, \ell^{q^{\prime}}\right.$ ), hence $f \notin\left(L^{p^{\prime}}, \ell^{q ;}\right) \quad[21$, Remark 2.4]. So the function $g$ defined by $\langle\phi, g\rangle=|\langle\phi, f\rangle|$ on $\left(\mathrm{L}^{\mathrm{q}^{\prime}}, \ell^{\mathrm{p}^{\prime}}\right)$ belongs to $\left(\mathrm{L}^{\mathrm{q}}, \ell^{\mathrm{p}}\right)$ and clearly to $\mathrm{P}\left(\mathrm{L}^{\mathrm{q}^{\prime}}, \ell^{\mathrm{p}^{\prime}}\right)$. But $\hat{\mathrm{g}} \notin\left(\mathrm{L}^{\mathrm{p}^{\prime}}, \ell^{\mathrm{q}^{\prime}}\right)$, otherwise $\hat{\mathrm{f}}$ would be in $\left(\mathrm{L}^{\mathrm{p}^{\prime}}, \ell^{q^{\prime}}\right)$. Therefore $g \notin R\left(\Phi_{\mathrm{pq}}\right)$. The remaining cases are similar

4. THE SPACE $R\left(\Phi_{\mathrm{pq}}\right)$ FOR $1 \leqq \mathrm{q}<2$.

We have seen that any measure in $R\left(\Phi_{p q}\right)(2 \leqq q \leqq \infty)$ is a linear combination of positive definite functions; we want to prove now that for $1 \leqq q<2$, any measure in $R\left(\Phi_{\mathrm{pq}}\right)$ is approximated by linear combinations of positive definite functions.

We endow the spaces $M$, and $\mathrm{L}_{1 \text { loc }}^{1}$ with the weak*-topology $\sigma\left(k 1, \mathrm{C}_{\mathrm{c}}\right)$, and $\sigma\left(\mathrm{L}_{10 \mathrm{l}}^{1}, \mathrm{~L}_{\mathrm{c}}^{1}\right)$ respectively [18, Chp. IV]. We consider $R\left(\Phi_{\mathrm{pq}}\right)$, and $R\left(\Phi_{\mathrm{pq}}\right)$ as subspaces of $\mathscr{A}$, and $\mathrm{L}_{1 \text { loc }}^{1}$ respectively.

PROPOSITION 4.1 . Let $1 \leqq p \leqq \infty, 1 \leqq q<2$. If there exists a measure $\nu \in M_{q},(\Gamma)$ if $\mathrm{p}=\infty, \nu \varepsilon\left(\mathrm{L}^{\mathrm{p}^{\prime}}, \mathrm{l}^{\mathrm{q}^{\prime}}\right)(\Gamma)$ if $\mathrm{p}<\infty$, such that $\stackrel{v}{\nu}$ is a measure and $\mu=1$ im $V^{\prime} * \psi_{U}$ in $M$, then $\mu \in R\left(\Phi_{\mathrm{pq}}\right)$ and $\hat{\mu}=\nu$. Conversely, if $\mu \varepsilon R\left(\Phi_{\mathrm{pq}}\right)$, then 1 im $\mu * \psi_{\mathrm{U}}=\mu$ in $M$.

PROOF. We prove the proposition for $p=\infty$, the proof for $p$ finite is the same.

For $\phi \varepsilon \Phi_{\infty 1}$, the net $\left\{\phi^{*} \psi_{U}\right\}$ converges to $\phi$ in $C_{c}[5]$, so we have tinat

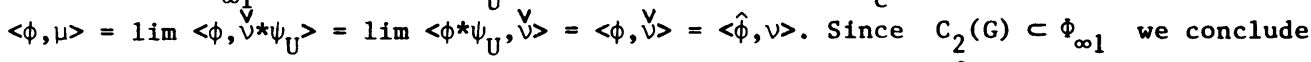
that $\mu$ is transformable and $\hat{\mu}=\nu$, so by Theorem $3.4, \mu \in R\left(\Phi_{\infty q}\right)$. The converse is clear.

REMARK 4.2. If $\mathrm{f} \in \mathrm{R}\left(\Phi_{\mathrm{pq}}\right)(1 \leqq \mathrm{p} \leqq \infty, 1 \leqq \mathrm{q}<2)$, then

$$
f * \psi_{U}(x)=\int_{G} \psi_{U}(x-t) f(t) d t=\int_{\Gamma} \hat{\psi}_{U}(\hat{x})[x, \hat{x}] d \hat{f}(\hat{x})
$$

because $f$ is transformable and $\hat{\psi}_{U} \varepsilon L^{1}(\hat{f})\left[5\right.$, Corollary 3.1]. Hence for $\phi \varepsilon c_{c}$ we have that

$$
\int_{G} \phi(x) f(x) d x=\lim \int_{G} \phi(x) \int_{\Gamma} \hat{\psi}_{U}(\hat{x})[x, \hat{x}] d \hat{f}(\hat{x}) d x
$$

This implies that $f(x)=\lim \int \hat{\psi}_{U}(\hat{x})[x, \hat{x}] d \hat{f}(\hat{x})$ where the limit exists on $L^{1}$ on any compact subset of G (c.f. [1, Theorem 9] and [7, Theorem 4.2]).

THEOREM 4.3. Let $1 \leqq q<2$. Every element in $R\left(\Phi_{p q}\right)$, hence in $R\left(\Phi_{p q}\right)$, can be approximated by elements in $\left\langle\mathrm{P}^{1}\left(\mathrm{~L}^{1}, \mathrm{l}^{\mathrm{P}^{\prime}}\right)\right\rangle$ if $2 \leqq \mathrm{p} \leqq \infty$, in $\left\langle\mathrm{P}\left(\mathrm{L}^{1}, \ell^{2}\right)\right\rangle$ if $1 \leq \mathrm{p} \leqq 2$.

PROOF. By Proposition 4.1 we only have to prove that for $\mu \in R\left(\Phi_{\infty q}\right)$, the net $\left\{\mu * \psi_{U}\right\}$ belongs to $\left\langle P\left(L^{1}, \ell^{P^{\prime}}\right)\right\rangle$.

By Theorem 3.4, the measure $\hat{\Omega}$ is a linear combination of positive measures $\mu_{j}$ 
$(j=1, \ldots, 4)$ in $M_{q^{\prime}}$. So by $[8,(2.5)]$ for $\phi \varepsilon \Phi_{\infty 1}$ we have that

$$
\begin{aligned}
\left|\left\langle\phi, \hat{\mu}_{j}^{*} \psi_{U}\right\rangle\right| & =\left|\left\langle\phi * \psi_{U}, \hat{\mu}_{j}\right\rangle\right|=\left|\left\langle\hat{\phi} \hat{\psi}_{U}, \mu_{j}\right\rangle\right| \leq\left.|| \mu_{j}||_{q^{\prime}}|| \hat{\psi}_{U}||_{\infty q}|| \hat{\phi}\right|_{\infty} \\
& \leq\left.\left.|| \mu_{j}||_{q} \cdot|| \hat{\psi}_{U}\right|_{\infty q}|| \phi\right|_{1} \quad(j=1, \ldots, 4)
\end{aligned}
$$

Since $\Phi_{\infty 1}$ is dense in $L^{1}\left[8\right.$, Proposition 2.5], we conclude that $\mu_{j} \star \psi_{U} \varepsilon L^{\infty}$ $(j=1, \ldots, 4)$. Now, if $f \in L^{1}(G)^{+}$, then by [8, P5] and the definition of the Fourier transform we have that $\left\langle f, \hat{\mu}_{j} * \psi_{U}\right\rangle=\left\langle f * \psi_{U}, \hat{\mu}_{j}\right\rangle=\left\langle\hat{f} \hat{\psi}_{U}, \mu_{j}\right\rangle \geqslant 0$, therefore $\hat{\mu}_{j} * \psi_{U} \varepsilon P(L)$ $(j=1, \ldots, 4)$, and we conclude that $\mu^{*} \psi_{U}$ belongs to $\left\langle P\left(L^{1}\right)>\right.$. If $\mu \varepsilon R\left(\Phi_{p q}\right)$, then each $\mu_{j}(j=1, \ldots, 4)$ belongs to $\left(L^{p^{\prime}}, l^{q^{\prime}}\right)$ and therefore $\left.\mu_{j} \hat{\psi}_{U} \varepsilon\left(L^{p^{\prime}}, l^{1}\right)[19, \S 7 h)\right]$, hence by (2.1) and the Hausdorff-Young theorem we have that $\hat{\mu}_{j} \psi_{U}$ is in $\left(L^{\infty}, \ell^{p}\right)$ if $2 \leqq \mathrm{p} \leqq \infty$, in $\left(\mathrm{L}^{\infty}, \ell^{2}\right)$ if $1 \leqq \mathrm{p} \leqq 2$. So for $\phi \varepsilon \mathrm{A}_{\mathrm{c}}^{+}$we have that

$\left\langle\phi, \hat{\mu}_{j} \psi_{U}\right\rangle=\left\langle\hat{\phi} \hat{\psi}_{U}, \mu_{j}\right\rangle \geq 0$, this implies by Theorem 2.5 that $\hat{\mu}_{j} \psi_{U}$ is in $P\left(L^{1}, \ell^{P^{\prime}}\right)$ if $2 \leqq \mathrm{p} \leqq \infty$, in $\mathrm{P}\left(\mathrm{L}^{1}, \ell^{2}\right)$ if $1 \leqq \mathrm{p} \leqq 2$.

REMARK 4.4. By Remark 2.3, Theorem 4.3 and Proposition 2.2 we conclude that the space $\left.\left\langle\rho_{\mathrm{c}}\right)\right\rangle$ is dense in $M_{\mathrm{T}}(\mathrm{c} . \mathrm{f} .[5, \S 4])$ and $\left\langle\mathrm{P}\left(\mathrm{C}_{\mathrm{c}}\right)\right\rangle$ is dense in $\mathrm{L}_{1 \mathrm{loc}} \cap M_{\mathrm{T}}$. PROPOSITION 4.5 . i) Let $1 \leqq q \leqq 2$. The spaces $\left\langle P\left(L^{1}\right)\right\rangle$ and $R\left(\Phi_{\infty q}\right)$ are dense in $R\left(\Phi_{\infty_{\mathrm{q}}}\right)$.

ii) Let $1<q \leqq 2$. The spaces $\left\langle P\left(L^{1}, \ell^{2}\right)\right\rangle$ and $R\left(\Phi_{2 q}\right)$ are dense in $R\left(\Phi_{2 q}\right)$. PROOF. In view of Theorem 4.3, we have to prove that $\left\langle P\left(L^{1}\right)\right\rangle \subset R\left(\Phi_{\infty q}\right)$ and $\left\langle\mathrm{P}\left(\mathrm{L}^{1}, \ell^{2}\right)>\subset \mathrm{R}\left(\Phi_{2 \mathrm{q}}\right)\right.$. First of all we recall (Remark 2.3) that $\mathrm{P}\left(\mathrm{L}^{1}\right) \subset M_{\mathrm{T}}^{\infty}$ and $\mathrm{P}\left(\mathrm{L}^{1}, \ell^{2}\right) \subset M_{\mathrm{T}}$.

Let $f \in P\left(L^{1}\right)$. By Theorem 2.5 and [6, Theorem II], its Fourier transform $\hat{f}$ belongs to $M_{q^{\prime}}$, hence $f \in R\left(\Phi_{\infty \eta}\right)$, since $f \in L_{10 c}^{1}$. If $f \in P\left(L^{l}, \ell^{2}\right)$, then again by Theorem 2.5 , f $\varepsilon\left(L^{q}, \ell^{2}\right)$ and by the Hausforff-Young theorem, $\hat{f} \varepsilon\left(L^{2}, \ell^{q^{\prime}}\right)$, hence f $\varepsilon R\left(\Phi_{2 q}\right)$.

We finally point out that for $2<\mathrm{p}<\infty$, the space $\left\langle\mathrm{P}\left(\mathrm{L}^{1}, \ell^{\mathrm{p}^{\prime}}\right)\right\rangle$ is not included in $R\left(\Phi_{\mathrm{pq}}\right)$, because as in [20, Theorem 5.5.1] using [21, Theorem 5] and [15, Theorems 5.6 and 15.9], we can prove that there exists a function $f$ in $\left(L^{\infty}, \ell^{p}\right)$ such that $\hat{f}$ is not a measure, hence the function $g$ defined by $\langle\phi, g\rangle=|\langle\phi, f\rangle|$ $\left(\phi \varepsilon\left(L^{1}, \ell^{P^{\prime}}\right)\right)$ belongs to $P\left(L^{1}, \ell^{P^{\prime}}\right)$ and $\hat{g}$ is not a measure, so $\hat{\mathbf{g}} \notin\left(L^{P^{\prime}}, \ell^{q^{\prime}}\right)$. Also, if $1 \leq \mathrm{p} \leqq \mathrm{q}<2$, then $\left\langle\mathrm{P}\left(\mathrm{L}^{1}, \ell^{2}\right)\right\rangle$ is not included in $R\left(\Phi_{\mathrm{pq}}\right)$. Indeed, let $\alpha=p^{\prime} / q^{\prime}+p^{\prime}$. Since $m$ is a inner measure there exists $I_{n} \subset[n, n+1)$ such that $m\left(J_{n}\right)=(1 / n)^{\alpha}$, where $J_{n}=\left\{x \in[n, n+1) \mid x \notin I_{n}\right\}$. So for each integer $n$ we define the function $f_{n}$ to be the product of $n$ times the characteristic function of the set $J_{n}$.

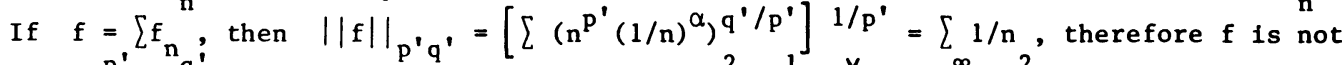
in $\left(L^{p^{\prime}}, \ell^{q^{\prime}}\right)$. Since each $f_{n}$ belongs to $\left(L^{2}, \ell^{1}\right), l_{n} \varepsilon\left(L^{\infty}, \ell^{2}\right)$, so by the Hausdorff-Young theorem $\left[f_{n}^{v}{ }^{n}\right.$ converges in $\left(L^{\infty}, l^{2}\right)$ to a function $f^{\prime}$, because $\left\|\sum \vec{f}_{n}^{v}\right\|_{\infty 2} \leqq \sum\left\|\vec{f}_{n}\right\|_{\infty 2} \leq \sum c|| f_{n} \|_{21}=\sum c n^{2}(1 / n)^{\alpha}=\sum c(1 / n)^{\alpha-2}<\infty$ since $\alpha>3$.

By the Lebesgue Convergence theorem and the fact that $S_{0}(R) \subset\left(L^{1}, \ell^{2}\right)$, for any $\phi$ in $S_{0}(R)$ we have $\langle\phi, \hat{f}\rangle=\langle\hat{\phi}, f\rangle=\left[\left\langle\hat{\phi}, f_{n}\right\rangle=\left[\left\langle\phi, f_{n}\right\rangle=\left\langle\phi, f^{\prime}\right\rangle\right.\right.$, hence $f^{\prime}=f^{v}$. Let 8 be the function on $\left(L, l^{2}\right)$ defined by $\langle\phi, g\rangle=|\langle\phi, \hat{f}\rangle|$. Clearly $g$ is in $P\left(L^{1}, l^{2}\right)$ and $\hat{g}$ is not in $\left(L^{p^{\prime}}, \ell^{q^{\prime}}\right)$ because for $\phi \varepsilon S_{0}(R)$ we have that $\langle\phi, \hat{g}\rangle=\langle\hat{\phi}, g\rangle=|\langle\hat{\phi}, f\rangle|=|\langle\phi, \hat{f}\rangle|$, this shows that $\hat{g} \varepsilon\left(L^{p^{\prime}}, l^{q^{\prime}}\right)$ iff $f \varepsilon\left(L^{p^{\prime}}, l^{q^{\prime}}\right)$. 
Therefore $\mathrm{g} \notin R\left(\Phi_{\mathrm{pq}}\right)$.

The construction of the function $f$ can be extended to $G$ using the partition of disjoint relatively compact subsets as in [7, §3]. Probably the same is true for $1 \leqq \mathrm{q}<\mathrm{p}<2$, but we were unable to decide the matter.

5. REPRESENTATION THEOREMS.

We will prove in this section (Theorem 5.4) that the representation theorems for $R\left(\Phi_{\infty 1}\right)$ and $P\left(C_{c}\right)$ in [1, Theorems 7 and 8] and [3, Theorems 4.1 and 4.2] respectively, hold for the space $L_{10 c}^{1} \cap R\left(\Phi_{p q}\right)(1 \leqq q<2,1 \leqq p \leqq \infty)$, and they are equivalent. We first give a remark easily deduce from [5, Theorem 3.3].

REMARK 5.1. Let $\mathrm{f} \in \mathrm{L}_{1 \mathrm{oc}}^{\mathrm{q}} \cap \mathrm{R}\left(\Phi_{\mathrm{pq}}\right)(1 \leqq \mathrm{p}, \mathrm{q} \leqq \infty)$.

i) If $\phi \in L^{1}(G)$, then $\phi^{*} f$ exists and $\hat{\phi} \in L^{1}(\hat{f})$. Therefore for locally almost a11 $x \in G$ we have that

$$
f * \phi(x)=\int_{G} f(y) \phi(x-y) d y=\int_{\Gamma} \hat{\phi}(\hat{x})[x, \hat{x}] d \hat{f}(\hat{x}) .
$$

ii) If the integral on the left is a continuous function of $x$ in a neighbourhood of 0 , then the formula in $i$ ) is valid for $x=0$. Hence under this hypothesis

$$
\int_{G} f(y) \phi(-y) d y=\int_{\Gamma} \hat{\phi}(\hat{x}) d \hat{f}(\hat{x}) \text {. }
$$

The next theorem includes [1, Theorem 3] as a particular case.

THEOREM 5.2. Let $1 \leqq \mathrm{p}, \mathrm{q} \leqq \infty$. If $\mathrm{f} \varepsilon \mathrm{L}_{1 \mathrm{oc}}^{\mathrm{q}} \mathrm{R}\left(\Phi_{\mathrm{pq}}\right)$, then

$$
\int_{G} f(x) \phi(x) d x=\int_{\Gamma} \hat{\phi}(-\hat{x}) d \hat{f}(\hat{x})
$$

for all $\phi \varepsilon \mathrm{L}_{\mathrm{c}}^{\mathrm{q}^{\prime}}$ such that $\hat{\phi} \varepsilon\left(\mathrm{L}^{\mathrm{p}}, \ell^{\mathrm{q}}\right)$ if $\mathrm{p}<\infty, \hat{\phi} \varepsilon\left(\mathrm{C}_{0}, \ell^{\mathrm{q}}\right)$ if $\mathrm{p}=\infty$.

PROOF. It is clear that the convolution $\phi^{*} \mathrm{f}$ exists for $\phi \varepsilon \mathrm{L}_{\mathrm{c}}^{\mathrm{q}^{\prime}}$ and $\mathrm{f} \varepsilon \mathrm{L}_{10 c}^{\mathrm{q}}$. If $\hat{\phi}$ is in either $\left(L^{p}, \ell^{q}\right)$ or $\left(C_{0}, \ell^{q}\right)$, then $\hat{\phi} \in L^{1}(\hat{f})$. So by our previous remark we have to prove that $f * \phi$ is continuous on a neighbourhood $U$ of 0 . Let $E$ be the support of $\phi$, and $s \in U$. If $1<q \leqq \infty$, then the map $x \longmapsto \phi_{x}$, where $\phi_{x}(y)=\phi(x-y)$, is continuous on G. So given $\varepsilon>0$ there exists a neighbourhood $V$ of 0 such that for all $x \in V$ we have that $\left\|\phi_{x}-\phi_{y}\right\|_{q^{\prime}}<\varepsilon /\left\|f X_{U-E}\right\|_{q}$, where $X_{U-E}$ is the characteristic function of $U-E$. So for $x \varepsilon U \cap V$ we have that

$|f * \phi(x)-f * \phi(s)| \leqq \int_{U-E}|f(y)|\left|\phi_{x}(y)-\phi_{s}(y)\right| d y \leqq\left|f X_{U-E}\right| q_{q}|| \phi_{x}-\phi_{y}|| q^{\prime}<\varepsilon$.

Therefore $f * \phi$ is continuous at $s$.

If $q=1$, then the map $x \longmapsto\left(f X_{U-E}\right)_{x}$ is continuous on $G$, and as before, there exists a neighbourhood of zero $V$ such that for $x$ in $U \cap V$ we have that $|f * \phi(x)-f * \phi(s)| \leqq\left\|\phi||_{\infty}||\left(f X_{U-E}\right)_{x}-\left(f \chi_{U-E}\right)_{s}\right\|_{1}<\varepsilon$. This ends the proof.

We need now to introduce Simon's generalization of Cesàro summability on locally compact abelian groups [22]. This consists of a family of functions $\left\{\phi_{U}\right\}$ (U being a compact neighbourhood of 0$)$ in $\left(C_{0}, \ell^{1}\right)$ with the following properties

$\phi_{\mathrm{U}} \geqq 0, \quad\left\|\phi_{\mathrm{U}}\right\|_{1} \leqq 1$

$\left\{\phi_{U}\right\}$ is an approximate identity for $L^{1}$

$\hat{\phi}_{U} \varepsilon C_{c}$ and $\underset{U}{\lim } \hat{\phi}_{U}(\hat{\mathbf{x}})=1$ for all $\hat{\mathbf{x}} \varepsilon \Gamma$.

The following representation theorem is an extension of [1, Theorem 7] (c.f. [3, 
Theorem 4.1]).

THEOREM 5.3. Let $1 \leqq \mathrm{q}<2,1 \leqq \mathrm{p} \leqq \infty$. If f $\varepsilon \mathrm{L}_{10 \mathrm{q}}^{\mathrm{q}} \mathrm{n} \mathrm{R}\left(\Phi_{\mathrm{pq}}\right)$, then (C. 1) $\int_{G} \underset{h}{h}(x) f(x) d x=\lim \int_{G} \breve{\phi}_{U}(x) \stackrel{v}{h}(x) f(x) d x=\int_{\Gamma} h(-\hat{x}) d \hat{f}(\hat{x})$

for all $h \in\left(L^{p}, \ell^{q}\right)(\Gamma)$ if $p<\infty, h \varepsilon\left(C_{0}, \ell^{q}\right)(\Gamma)$ if $p=\infty$.

Furthermore if $r=2 q /(2 q-1)$ and $2 \leqq p \leqq \infty$, then

$$
\iint f(x-y) \phi(x) \overline{\psi(y)} d x d y=\int_{\Gamma} \hat{\phi}(\hat{x}) \overline{\hat{\psi}(\hat{x})} d \hat{f}(\hat{x})
$$

for all $\phi, \psi$ in $\left(\mathrm{L}^{\mathrm{r}}, \ell^{\mathrm{p}^{\prime}}\right)$. The double integral exists not necessarily as a Lebesgue integral but as the sum of the convergent series

$$
\sum_{\alpha} \sum_{\beta} \int_{v_{\alpha}} \int v_{\beta} f(x-y) \phi(x) \overline{\psi(y)} d x d y
$$

where $v_{\alpha}, v_{\beta}$ are finite union of the sets $L_{\alpha}$, as $\underset{v}{d e f i n e d}$ in $[7, \S 3]$.

PROOF. Suppose $p<\infty$. If $h \in\left(L^{p}, \ell^{q}\right)(\Gamma)$, then $h$ belongs to either ( $\left.L^{q^{\prime}}, \ell^{p^{\prime}}\right)(G)$ if $1 \leqq p \leqq 2$, or $\left(L^{q^{\prime}}, l_{v v}^{2}\right)(G)$ if $p>2$. Since $\left\{\phi_{U}\right\} \subset C_{c}(G)$, we have that $h \phi_{U} \varepsilon L_{c}^{q^{\prime}}$ and therefore $h * \phi_{U}=\left(h \phi_{U}\right) \wedge$. So by Theorem 5.2 we conclude that

$$
\int_{G} \oint_{U}(x) \stackrel{v}{h}(x) f(x) d x=\int_{\Gamma} h * \phi_{U}(-\hat{x}) d \hat{f}(\hat{x}) .
$$

Since $\hat{\mathrm{f}} \varepsilon\left(\mathrm{L}^{\mathrm{p}^{\prime}}, \ell^{\mathrm{q}^{\prime}}\right)$ and $\lim \mathrm{h} * \phi_{U}=\mathrm{h}$ in $\left(\mathrm{L}^{\mathrm{p}}, \ell^{\mathrm{q}}\right)$ [8, Proposition 1.8] the integral on the right converges to $\int h(\hat{x}) d \hat{f}(\hat{x})$ and this proves the first part of the theorem.

Let $\phi, \psi$ in $L_{c}^{r}$. Since $r=2 q /(2 q-1)$ and $1 \leq q<2$, we have that $1 / r=1-1 / 2 q$, hence $1 / 2 \leq 1 / \mathrm{r} \leqq 3 / 4$ and therefore $1<\mathrm{r}<2$. If $\phi \varepsilon \mathrm{L}_{\mathrm{c}}^{\mathrm{r}}$, then $\hat{\phi} \varepsilon\left(\mathrm{C}_{0}, l^{\mathrm{r}^{\prime}}\right)$ and $\left(\phi^{*} \psi\right)^{\wedge} \varepsilon\left(\mathrm{C}_{0}, \ell^{\mathrm{q}}\right)$ because $\mathrm{r}^{\prime}=2 \mathrm{q} \quad[12, \S 7]$. So by Theorem 5.2

$$
\iint \mathrm{f}(\mathrm{x}-\mathrm{y}) \phi(\mathrm{x}) \overline{\psi(\mathrm{y})} \mathrm{dx} d \mathrm{y}=\int \hat{\phi}(\hat{\mathbf{x}}) \overline{\hat{\psi}(\hat{\mathbf{x}})} \mathrm{d} \hat{\mathrm{f}}(\hat{\mathrm{x}}) .
$$

Set $B(\phi, \psi)$ to be equal to the left side of (5.4). So by Holder's inequality $|B(\phi, \psi)| \leq\left.|| \hat{\mathrm{f}}\right|_{\mathrm{p}^{\prime} \mathrm{q}^{\prime}}|| \hat{\phi} \hat{\psi}\left\|_{\mathrm{pq}} \leqq|| \hat{\mathrm{f}}\right\|_{\mathrm{p}^{\prime} \mathrm{q}^{\prime}}|| \hat{\phi}\left\|_{\mathrm{pr}}{ }^{\prime}|| \hat{\psi}\right\|_{\mathrm{pr}}$, therefore $|\mathrm{B}(\phi, \psi)| \leqq \mathrm{C}|| \psi \|\left._{\mathrm{rp}} \cdot|| \phi\right|_{\mathrm{rp}}, \quad$ if $1 \leqq \mathrm{p} \leqq 2$ and $|\mathrm{B}(\phi, \psi)| \leqq \mathrm{C}|| \psi \|_{\mathrm{r} 2}|| \phi||_{\mathrm{r} 2}$ if $\mathrm{p}>2$, where $C$ is a constant depending on $f, p$ and $q$.

If $g \varepsilon\left(L^{r}, \ell^{p^{\prime}}\right)$, then $\|g\|_{r p^{\prime}}=\left(\sum\left\|g_{\alpha}\right\|_{r}^{p^{\prime}}\right)^{1 / p^{\prime}}$ where $g_{\alpha}=g x_{L_{\alpha}}$. So for $\phi, \psi$

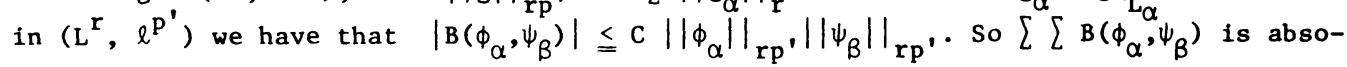
lutely convergent and the left side of (5.4) exists as stated in the theorem.

Finally, since $\sum g_{\alpha}$ converges in the norm of $\left(L^{r}, l^{p^{\prime}}\right)$ to $g$ we have that $\sum \sum B\left(\phi_{\alpha}, \psi_{\beta}\right)=\int \hat{\phi}(\hat{x}) \overline{\hat{\psi}(\hat{x})} \mathrm{d} \hat{f}(\hat{x})$. The proof for $2<p \leqq \infty$ is similar.

THEOREM 5.4. If $1 \leqq \mathrm{q}<2,1 \leqq \mathrm{p} \leqq \infty$ and $\mathrm{f} \varepsilon \mathrm{L}_{\text {loc }}^{\mathrm{q}}$, then the following are equivalent

i) $\mathrm{f} \in \mathrm{R}\left(\Phi_{\mathrm{pq}}\right)$

ii) There exists a unique $\mu$ in $M_{q^{\prime}}(\Gamma)$ if $p=\infty$, in $\left(L^{p^{\prime}}, \ell^{q^{\prime}}\right)(\Gamma)$ if $p<\infty$, such that for all $\phi, \psi$ in $\left(L^{r}, \ell^{p^{\prime}}\right)(G)$, where $r=2 q /(2 q-1)$,

$$
\iint f(x-y) \phi(x) \overline{\psi(y)} d x d y=\int \hat{\phi}(\hat{x}) \overline{\hat{\psi}(\hat{x})} d \mu(\hat{x})
$$

the double integral exists as in Theorem 5.3.

iii) There exists a unique measure $\mu$ as in part ii) such that 


$$
f(x)=\lim \int_{\Gamma} \hat{\psi}_{U}(\hat{x})[x, \hat{x}] d \mu(\hat{x})
$$

where the 1imit exists as in Remark 4.2.

PROOF. By Theorem 5.3 part i) implies part ii) and by Proposition 4.1 part iii) implies part i).

Suppose that ii) holds. Since $\psi_{U}=\beta_{U} * \tilde{\beta}_{U}$ and $\beta_{U} \varepsilon L_{c}^{2}$, for all $t$ in $G$ we have

$$
\iint f(x-y) \beta_{U}(x-t) \overline{\beta_{U}(y)} d \mathbf{x} d y=\int[t, \hat{\mathbf{x}}] \hat{\beta}_{U}(\hat{\mathbf{x}}) \overline{\hat{\beta}_{U}(\hat{\mathbf{x}})} d \mu(\hat{\mathbf{x}}) \text {. }
$$

The left side is equal to

$$
\int f(x) \beta_{U} \star \tilde{\beta}_{U}(t-x) d x=\int f(x) \psi_{U}(t-x) d x=f * \psi_{U}(t),
$$

the right side is equal to $\int \hat{\psi}_{U}(\hat{x})[t, \hat{x}] d \mu(\hat{x})$, and $\psi_{U}{ }^{*} f$ converges to $f$ in the sense of part iii). Hence we conclude that ii) implies iii).

6. FURTHER RESULTS.

In this last section we want to give a characterization of the set of Fourier multipliers from the space $\Phi_{\infty \mathrm{q}}(1 \leq \mathrm{q} \leq \infty)$ to $L^{1}$ and $M_{1}$. This will allow us to extend Dupuis' characterization theorem [6, Theorem III].

Following the notation in [23] we denote by $M\left(\Phi_{\infty_{q}}\right) \quad\left(M\left(\Phi_{\infty q}\right)\right)$ the space of Fourier multipliers from $\Phi_{\infty_{\mathrm{q}}}$ to $\mathrm{L}^{1}\left(M_{1}\right)$, that is, $M\left(\Phi_{\infty_{\mathrm{q}}}\right)\left(M\left(\Phi_{\infty_{\mathrm{q}}}\right)\right)$ is the space of all functions (measures) $f$ on $\Gamma$ such that $f \hat{\phi}$ is in $L^{1}\left(M_{1}\right)$ for all $\phi \varepsilon \Phi_{\infty q}(G)$.

Since $\Phi_{\infty \mathrm{q}}=\mathrm{C}_{\mathrm{c}}$ for $2 \leqq \mathrm{q} \leqq{ }^{\infty}$, we consider the characterization of these spaces for $1 \leqq q \leqq 2$.

THEOREM 6.1. Let $1 \leqq q \leqq 2$. Then $M\left(\Phi_{\infty_{q^{\prime}}}\right)=\left(L^{1}, \ell^{q^{\prime}}\right)$ and $M\left(\Phi_{\infty q^{\prime}}\right)=M_{q^{\prime}}$. PROOF. By the Young's inequality $\left(L^{1}, l^{q^{\prime}}\right) \subset M\left(\Phi_{\infty q}\right)$ and $M_{q^{\prime}} \subset M\left(\Phi_{\infty q}\right)$.

Let $\mu \varepsilon M\left(\Phi_{\infty \mathrm{q}}\right)$ and suppose that $\lim \phi_{\mathrm{n}}=\phi$ in $\Phi_{\infty \mathrm{q}}$ and $\lim \mu \hat{\phi}_{\mathrm{n}}=\mathrm{h}$ in $M_{1}$, so for $f \in C_{c}$ we have that

$$
\begin{aligned}
|<\mathrm{f}, \mu \hat{\phi}-\mathrm{h}>| & \leqq\left|<\mathrm{f}, \mu \hat{\phi}_{\mathrm{n}}-\mu \hat{\phi}>\right|+\left|<\mathrm{f}, \mu \hat{\phi}_{\mathrm{n}}-\mathrm{h}>\right| \leq\left|<\mathrm{f}\left(\hat{\phi}_{\mathrm{n}}-\hat{\phi}\right), \mu>\right|+|| \mathrm{f}||_{\infty}|| \mu \hat{\phi}_{\mathrm{n}}-\left.\mathrm{h}\right|_{1} \\
& \leq \mathrm{C}|| \mathrm{f}||_{\infty}|| \hat{\phi}_{n}-\hat{\phi}||_{\infty \mathrm{q}}+|| \mathrm{f}||_{\infty}|| \mu \hat{\phi}_{\mathrm{n}}-\mathrm{h}||_{1}
\end{aligned}
$$

where $C$ is a constant depending on the support of $f$. Since $C_{c}$ is dense in $C_{0}$, we conclude by the Closed Graph theorem that the map $\phi \longmapsto \mu \hat{\phi}$ from $\Phi_{\infty q}$ to $M_{1}$ is continuous. Hence by Remark 3.2, $\mu \in M_{q^{\prime}}(\Gamma)$. If $f \in M\left(\Phi_{\infty q}\right)$, then the measure $|\mathbf{f}| d m b e-$ longs to $M_{q^{\prime}}(\Gamma)$ and therefore $f \in\left(L^{1}, \ell^{q^{\prime}}\right)(\Gamma)$.

From Theorem 6.1 we see that $M\left(C_{c}\right)=\left(L^{1}, \ell^{2}\right)$, as proven in [23], and $M\left(C_{c}\right)=M_{2}$. We write $F \Phi_{\infty_{q}}$ to denote the set $\left\{\hat{\phi} \mid \phi \varepsilon \Phi_{\infty_{q}}\right\}$.

THEOREM 6.2. Let $1 \leq q \leq 2$. Then $P\left(F \Phi_{\infty q}\right)=\left(L, l, l q \cap \rho\right.$ and $\left(F \Phi_{\infty q}\right)=M_{q}, \cap \rho$. Hence $P\left(F \Phi_{\infty_{q}}\right)=P\left(C_{0}, \ell^{q}\right)$ and $\rho\left(F \Phi_{\infty q}\right)=\rho\left(C_{0}, \ell^{q}\right)$.

PROOF. By [6. Proposition IV] and Theorem 6.1, $P\left(F \Phi_{\infty q}\right) \subset\left(L^{1}, \ell^{q^{\prime}}\right) \cap \rho$ and $\rho\left(F \Phi_{\infty q}\right) \subset M_{q}, \cap \rho$.

Take $\mu^{q} \varepsilon M_{q^{\prime}} \cap \rho$ and $\hat{\phi} \varepsilon\left(F \Phi_{\infty q}\right)^{+}$. Since $\left\{\hat{\phi}^{*} e_{\alpha}\right\}$ converges to $\hat{\phi}$ in $\left(C_{0}, l^{q}\right)$, $\hat{\phi}^{*} e_{\alpha} \varepsilon A_{c}$ and $\left(\hat{\phi}^{*} e_{\alpha}\right)^{v}=\phi_{e_{\alpha}}^{v} \geq 0$, we have that $\hat{\phi}, \mu=1$ im $\hat{\phi}^{*} e_{\alpha}, \mu \geqq 0$. Therefore $\mu \varepsilon f\left(F \Phi^{\infty}\right)$. Since $\left(L^{1}, \ell^{q^{\prime}}\right)$ is included in $M_{q^{\prime}}[8,(1.9)]$ we conclude that $\left(L^{1}, \ell^{q^{\prime}}\right) \cap \rho \subset P\left(F \Phi_{\infty}\right)$. The last equality follow from Remark 2.3 and an argument like that of Theorem 2.5. 


\section{REFERENCES}

1. HOLLAND, F. On the Representations of Functions as Fourier Transforms of Unbounded Measures. Proc. London Math. Soc. (3) 30 (1975), 347-365.

2. COOPER, J.L.B. Positive Definite Functions of a Real Varialble. Proc. Londen Math. Soc. (3) 10 (1960), 53-66.

3. STEWART, J. Unbounded Positive Definite Functions. Can J. Math. 21 (1969), 1309-1318.

4. BOCHNER, S. Vorlesungen über Fouriersche Integrale. Akademische Verla-Sgesellschaft, Leipzig, 1932.

5. ARGABRIGHT, L. and GIL DE LAMADRID, J. Fourier Analysis of Unbounded Measures on Locally Compact Abelian Groups. Memoirs Am. Math. Soc. 145, 1974.

6. DUPUIS, C. Quasimeasures de Type Positif. Bul1. Sc. Math, 2-e serie 105 (1981) 169180.

7. STEWART, J. Fourier Transforms of Unbounded Measures. Can. J. Math, 31 (1979), 1281-1292.

8. TORRES DE SQUIRE, M. Multipliers for Amalgams and the Algebra S (G). To appear in Can. J. Math.

9. FEICHTINGER, H.G. On a New Segal Algebra. Mh. Math. 22 (1981) 269-289.

10. FEICHTINGER, H.G. Un Espace de Banach de Distributions Temperèes sur les Groups Localement Compacts Abeliens. CR. Acad. Sci. Paris 290 (1980) serie A, 791-794.

11. BoURBAKI, N. Elements de Mathématique. Integration. Chps. 1-4. Act. Sci. et Ind. 1175, Hermann et Cie., Paris, 1973.

12. BERTRANDIAS, J.P. and DUPUIS, C. Transformation de Fourier sur les Espaces $\ell^{\mathrm{P}}\left(\mathrm{L}^{\mathrm{P}^{\prime}}\right)$. Ann. Inst. Fourier (Grenoble) 29 (1979), 189-206.

13. LOSERT, V. A Characterization of the Minimal Strongly Character Invariant Segal Algebra. Ann. Inst. Fourier (Grenoble) 30 (1980), 129-139.

14 COWLING, M. Some Applications of Grothendiek's Theory of Topological Tensor Products in Harmonic Analysis. Math. Ann. 232 (1978), 273-285.

15. TORRES DE SQUIRE, M. Amalgams of $\mathrm{L}^{\mathrm{p}}$ and $\ell^{\mathrm{q}}$. $\mathrm{Ph}$. D. Thesis, McMaster University, Canada, 1984.

16. EBERLEIN, W.F. Characterizations of Fourier-Stieltjes Transform. Duke Math. J. 22 (1955), 465-468.

17. TORRES DE SQUIRE, M. Local Complements to the Hausdorff-Young Theorem for Amalgams. Preprint.

18. BOURBAKI, N. Espaces Vectoriels Topologiques. Chps. 3-5. Act. Sci. et Ind. 1229, Hermann et Cie., Paris, 1967.

19. BERTRANDIAS, J.P., DATRY, C. and DUPUIS, C. Unions et Intersections d'Espaces $L^{\text {P }}$ Invariantes par Translation ou Convolution. Ann. Inst. Fourier (Grenoble) 28 (1978), 53-84.

20. LARSEN, R. An Introduction to the Theory of Multipliers. Grundl. Math. Wiss. 175, Speinger-Verlag, Berlin/New York, 1971.

21. HOLLAND, F. Harmonic Analysis on Amalgams of $\mathrm{L}^{\mathrm{p}}$ and $\mathrm{L}^{\mathrm{q}}$. J. London Math. Soc. (2) 10 (1975), 295-305.

22. SIMON, A.B. Cesaro Summability on Groups: Characterization and Inversion of Fourier Transforms. Function Algebras. Proc. Internat. Sympoc. on Function Algebras, Tulane University, 1965.

23. EDWARDS, R.E., HEWITT, E. and RITTER, G. Fourier Multipliers for certain Spaces of Functions with Compact Supports. Invent. Math. 40 (1977), 37-57. 


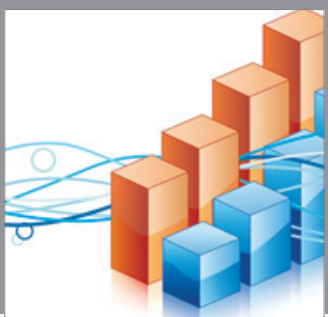

Advances in

Operations Research

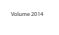

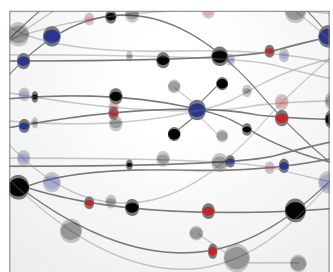

\section{The Scientific} World Journal
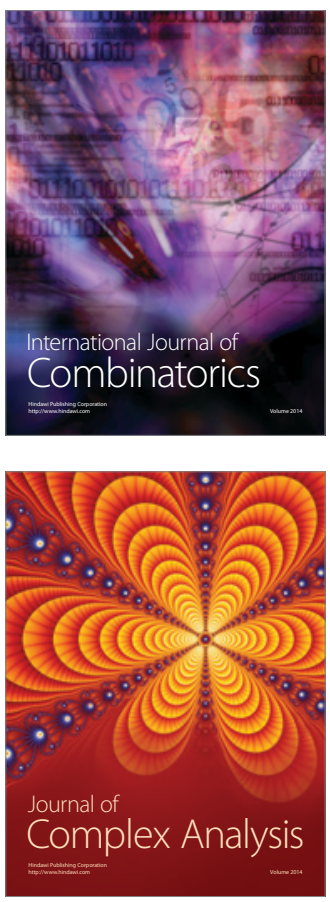

International Journal of

Mathematics and

Mathematical

Sciences
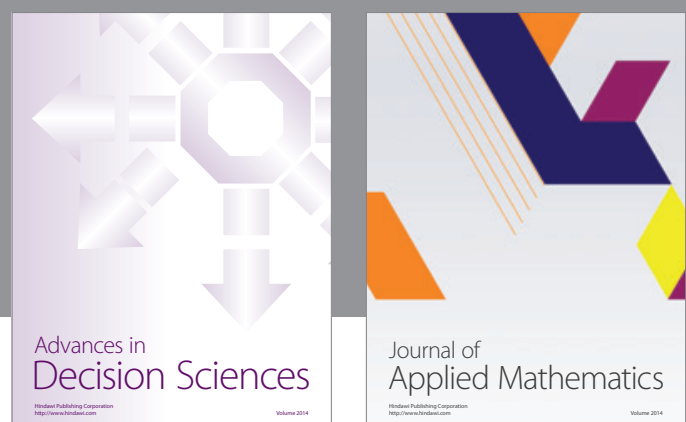

Journal of

Applied Mathematics
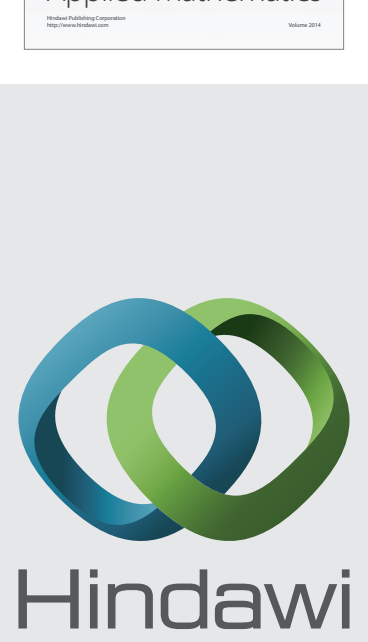

Submit your manuscripts at http://www.hindawi.com
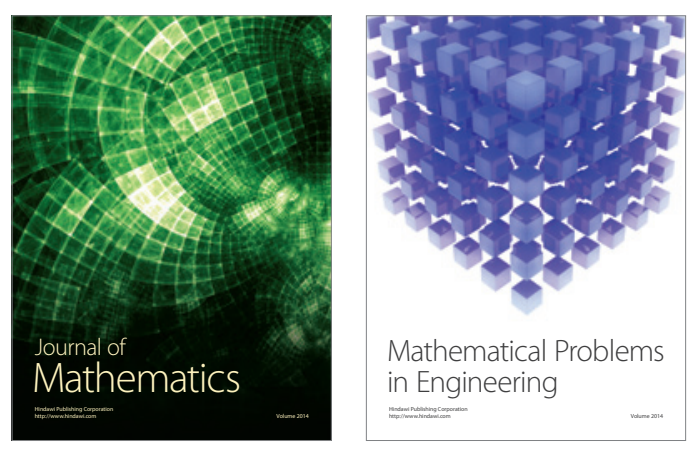

Mathematical Problems in Engineering
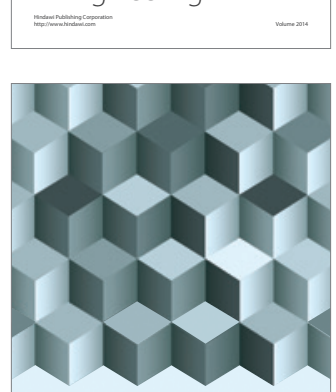

Journal of

Function Spaces
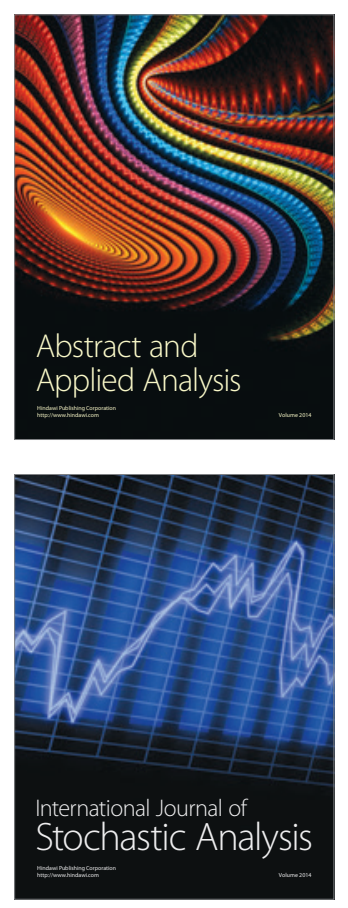

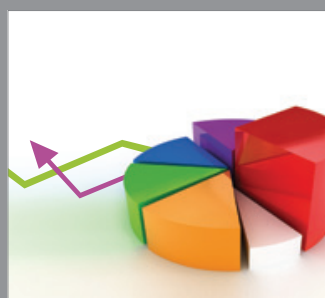

ournal of

Probability and Statistics

Promensencen
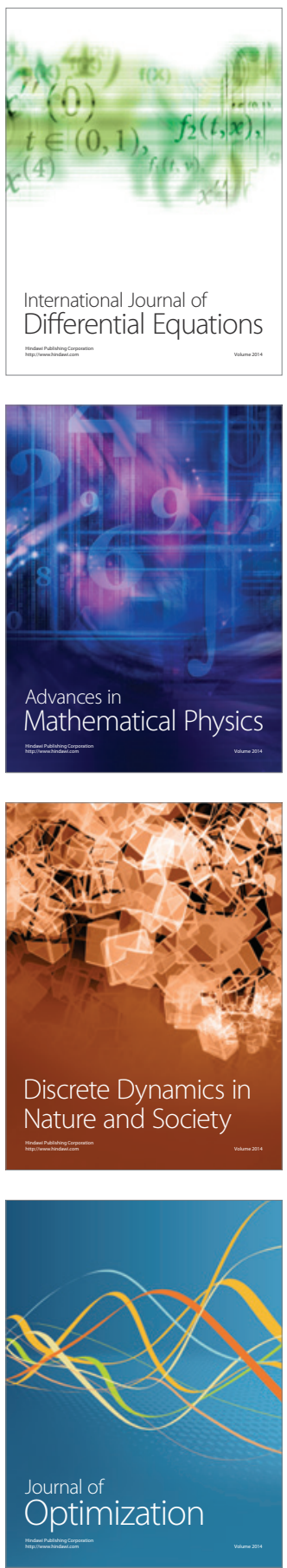